\author{
Alain (Georges) Leduc \\ École supérieure d'Art de Lorraine/Metz (ÉSAL), France \\ http://dx.doi.org/10.18778/8088-896-8.06
}

\title{
LA DOMINATION DES CERVEAUX ET DES CORPS
}

Toute lecture serait-elle désormais impossible de Sade, d'Octave Mirbeau, de Roger Vailland ? Athées, laïques, matérialistes sont-ils devenus une minorité ostracisée, dans cette Europe qui se disloque actuellement et part pitoyablement en miettes sous nos yeux? Il n'en est plus aujourd'hui, France, Irlande (où un enfant ne peut être admis dans l'éducation publique s'il n'a pas été baptisé), Pologne, Portugal, Malte, etc. (qui combattent obscènement contraception et avortement), que pour le débat public et médiatique entre seuls adeptes et représentants des trois religions monothéistes, dites « révélées ».

Confrontés à la régression de la pensée, aux préjugés qui se multiplient, il nous faut défendre l'héritage des Lumières, leurs valeurs, contre l'obscurantisme en plein renouveau : les valeurs de la raison, de la connaissance, de l'éducation.

Nous nous appuierons sur trois de leurs descendants directs, des théoriciens résolument athées et matérialistes. L'un épigone immédiat; le deuxième à la charnière de deux siècles, le $\mathrm{XIX}^{\mathrm{e}}$ et le $\mathrm{XX}^{\mathrm{e}}$; le troisième mort voici une cinquantaine d'années, en 1965. Il s'agit respectivement de Sade, d'Octave Mirbeau et de Roger Vailland.

Resucées et prolongateurs d'acquis antiques (Épicure, Diogène Laërce, Lucrèce, qui a fait de l'atomisme épicurien un sublime poème), le Polonais Nicolas Copernic (1473-1543), avec l'héliocentrisme, l'Italien Galilée (1564-1642) Eppur si muove!, Giordano Bruno (1548-1600), naturellement - brûlé vif en place publique par les séides du Vatican -, étendirent les frontières de la science jusqu'au XVII ${ }^{e}$ siècle. Les Lumières débusquèrent leurs premières lueurs dans le spinozisme.

Enfin Voltaire vint. L'affaire Calas ne fut pas son seul grand combat :

Voyez comme il s'engage, en 1766, pour défendre ce malheureux chevalier de La Barre. Âgé de 20 ans, le jeune homme a été torturé, a eu la langue tranchée, puis a été décapité et brûlé parce qu'il n'avait pas enlevé son chapeau devant une procession, chantait des chansons « impies » et lisait... le Dictionnaire philosophique de Voltaire ${ }^{1}$.

\footnotetext{
1 Philippe Sollers. Propos recueillis par Frédéric Joignot, Le Monde du 9 avril 2015.
} 
Mais il y a libertins et libertins. Le mot « libertin », nous l'apprenions au lycée (tout ceci ne s'enseigne plus maintenant), possède deux sens, selon qu'on l'utilisait au XVII ${ }^{\mathrm{e}}$ siècle ou au siècle suivant, qu'il induit prioritairement la question de l'affranchissement vis-à-vis de Dieu ou plutôt la « liberté » sexuelle. Le libertin, c'est d'abord l'homme (ou la femme) qui se libère progressivement de l'idée-de-Dieu-que-l'on-a-dans-la-tête, puis c'est l'homme (ou la femme) sans Dieu. Pas la seule inconduite de l'aristocrate s'estimant au dessus des lois, qui veut qu'on chasse la femme comme on chasse le lièvre, la traque, et aille jusqu'au couperet - Valmont, chez Laclos, Don Giovanni, chez Da Ponte et Mozart. Les libertins, chez Pascal, sont déjà des orphelins de Dieu. Valmont est désinvolte. Son élégance, son insolence, lui sont naturelles. Dieu ? On a décapité un roi - de droit divin $($ sic $)$ - et Dieu n'a pas bougé d'un iota. Dieu n'existe pas.

Rappelez-vous donc dans Candide $(1759)^{2}$ : Pangloss arrive à Constantinople et entre dans une mosquée. Là, il croise « un vieil imam » et « une jeune dévote, très jolie, qui disait ses patenôtres ». La jeune femme laisse tomber son bouquet, Pangloss le lui rend « avec un empressement respectueux $\gg$ quand l'imam s'aperçoit qu'il est chrétien. Il est aussitôt condamné « à cent coups de latte sur la plante des pieds » et « envoyé aux galères ». Dans sa pièce Le Fanatisme ou Mahomet (1741), Voltaire, qui a toujours eu un sens aigu de la formule assassine, n'hésite pas à mettre en scène le prophète qui déclare : « Il faut un nouveau culte, il faut de nouveaux fers; il faut un nouveau dieu pour l'aveugle univers ». Il s'en prend aussi en sous-main à la religion catholique. Car il se méfie de toutes les religions. Dans son Traité sur la tolérance (1763), devenu un best-seller inattendu au lendemain des attentats du 11 janvier (2015), comme Paris est une fête, d'Hemingway, le sera après ceux du 13 novembre (2015 également) ${ }^{3}$, il tranche à leur propos : «Elles ont toutes le même bandeau sur les yeux quand il faut incendier les villes et les bourgs de leurs adversaires $\gg$. Il décrit les crises de folie causées par la foi : « Je les ai vus ces convulsionnaires, je les ai vus tordre leurs membres et écumer. Ils criaient : "Il faut du sang !" » Il est à ce point désarmé par les fous-de-Dieu qu'il se demande : «Que répondre à un homme qui vous dit qu'il aime mieux obéir à Dieu qu'aux hommes, et qui en conséquence est sûr de mériter le ciel en vous égorgeant ?»

2 Cité par Ph. Sollers, « Il manque, Voltaire, là ! », Le Monde, 11 avril 2015, suppl. $\ll$ Culture et idées $»$, p. 4.

3 Une ruée dans les librairies a fait passer le classique des Lumières de 11500 exemplaires en 2014 à 185000 exemplaires vendus fin 2015. Quant aux souvenirs de l'écrivain nord-américain, ils se sont vendus à raison de 28000 exemplaires par semaine dans les deux mois qui suivirent l'attentat dit « des terrasses », contre 100 quelques jours auparavant. 
La question de Leibniz (1646-1716) - « Pourquoi y a-t-il quelque chose plutôt que rien? »- n'a pas moins de vigueur pour une démarche scientifique que son inverse - « Pourquoi n'y aurait-il rien, pourquoi l'univers n'aurait-il pas toujours été ? $\gg^{4}$ Le hasard, un grand ordinateur, un créateur suprême ? Le «Dieu (qui) ne joue pas aux dés », d'Einstein ou le « grand horloger » de Voltaire?

Avec Voltaire, Sade, la France est aux avant-feux.

Pierre-Simon de Laplace (1749-1827) est célèbre pour une boutade par laquelle, devant Napoléon Bonaparte, il aurait relégué Dieu au rang de supposition. Comme il présentait au général la première édition de son Exposition du Système $d u$ monde, celui-ci lui aurait dit : « Newton a parlé de Dieu dans son livre. J'ai déjà parcouru le vôtre et je n'y ai pas trouvé ce nom une seule fois ». À quoi Laplace aurait répondu : «Citoyen Premier consul, je n’ai pas eu besoin de cette hypothèse $\gg$. Variante : feuilletant la Mécanique céleste $e^{5}$, Napoléon fit remarquer à Laplace qu'il n'y était nulle part fait mention de Dieu. Laplace aurait alors répondu à Napoléon : « Dieu est une jolie hypothèse qui explique bien des choses. Si cette hypothèse explique tout, elle ne permet de prédire rien et n'entre donc pas dans mon domaine d'étude $\gg^{6}$.

Il est singulier de remarquer que, parmi les Lumières ou leurs descendants directs, ce soit Sade, après Diderot ${ }^{7}$ bien sûr, puis dans le sillage de La Mettrie, L'Homme machine 8 qui ait poussé le plus loin la question du matéria2010.

${ }^{4}$ S. Huet, « Hawking versus Dieu », Libération, suppl. « Sciences », 16 septembre

${ }^{5}$ Sa Mécanique céleste est publiée en cinq volumes. Les deux premiers, publiés en 1799, contiennent les méthodes pour calculer les mouvements des planètes, pour déterminer leurs formes et pour résoudre les problèmes liés aux marées. Le troisième et le quatrième, publiés respectivement en 1802 et en 1805, contiennent les applications de ces méthodes et diverses tables astronomiques. Le cinquième volume (1825) est principalement historique mais il fournit en appendice les résultats de ses dernières recherches.

${ }^{6}$ Wikipedia, notice Pierre-Simon de Laplace : https://fr.wikipedia.org/wiki/ Pierre-Simon_de_Laplace Consulté le 4 septembre 2017.

${ }^{7}$ La plus grande originalité de Diderot réside sans doute dans sa conception hardie d'une sensibilité de la matière qu'il ne peut imaginer inerte. Il soutient, en particulier dans Le Rêve de d'Alembert (1769), que toutes les molécules sont animées de sensations et de pensées, plus ou moins élaborées selon les cas.

${ }^{8}$ L'Homme Machine, de Julien Offray de La Mettrie publié à Leyde (en raison de la censure sévissant en France) en 1748. 
lisme. Une logique dont le point d'orgue sera chez lui avec des textes comme son Dialogue entre un prêtre et un moribond ou La philosophie dans le boudoir (1795). Le Dialogue entre un prêtre et un moribond est un court ouvrage écrit en prison par le marquis de Sade en 1782, et qui n'a été pour la première fois édité qu'en $1926^{\circ}$. Dans ce dialogue philosophique, celui-ci y affirme son libertinage et son athéisme à travers le moribond qui refuse de se repentir. Ce dernier, athée, s'oppose au prêtre qui tente de lui faire admettre la nécessité de l'existence de Dieu. Le moribond, lui, insiste au contraire sur l'impossibilité de prouver rationnellement cette existence; il développe une pensée rationnelle et matérialiste, sur laquelle il fait reposer la morale et la justice humaine.

«Ce ne sont pas les gens qui lisent Sade qui ont fait les camps de concentration. Ce sont des gens qui n'avaient jamais lu Sade $\gg^{10}$, écrit Dominique Aury, l'auteur d'Histoire d'O (1954).

Aux yeux de Sade, tout se vaut. Mais quelle « valeur », au sens positif, porterait la religion ? Nous pourrions forger ce mot, sur le bien oublié verbe « dévaloir ». Tout se dévaut/dévot. Chez Sade, les répliques sont cinglantes, au fleuret ; ainsi :

Le moribond - [...] Perfectionne ta physique et tu comprendras mieux la nature, épure ta raison, bannis tes préjugés et tu n'auras plus besoin de ton dieu.

Le prêtre - Malheureux ! je ne te croyais que socinien ${ }^{11}$, j'avais des armes pour te combattre, mais je vois bien que tu es athée. [...]

Le moribond $-[\ldots]$ Tu ajoutes erreurs sur erreurs, moi je les combats toutes. Lequel de nous deux est aveugle ?

Le prêtre - Vous ne croyez donc point en Dieu ?

Le moribond $-[\ldots]$ Mon ami, prouve-moi l'inertie de la matière, et je t'accorderai le créateur, prouve-moi que la nature ne se suffit pas à elle-même, et je te permettrai de lui supposer un maître. [...] Je crois le soleil parce que je le vois, je le conçois comme le centre de réunion de toute la matière inflammable de la nature, sa marche périodique me plaît sans m'étonner. C'est une opération de physique, peut-être aussi simple que celle de l'électricité, mais qu'il ne nous est pas permis de comprendre.

Le moribond $-[\ldots]$ Si j'étais assez faible que de me laisser surprendre à tes ridicules systèmes sur l'existence fabuleuse de l'être qui me rend la religion nécessaire, sous quelle forme me conseillerais-tu de lui offrir un culte? Voudrais-tu que

9 D. A. F. de Sade, Dialogue entre un prêtre et un moribond, Paris, Gallimard, coll. « La Pléiade », 1990.

10 D. Aury, «Une fille amoureuse », [in] D. Aury, Retour à Roissy, Le Livre de poche, Paris, 1975.

11 Adepte du socinianisme, un courant chrétien remontant à l'Italien Fausto Socin - alias Fausto Sozzini (1539-1604), qui refusait la doctrine chrétienne de la Trinité, et se présentait comme libéral. 
j'adoptasse les rêveries de Confucius, plutôt que les absurdités de Brahma, adorerais-je le grand serpent des nègres, l'astre des Péruviens ou le dieu des armées de Moïse, à laquelle des sectes de Mahomet voudrais-tu que je me rendisse, ou quelle hérésie de chrétiens serait selon toi préférable ? ${ }^{12}$

Et d'ajouter (il n'y a pas de « prophète », de « Dieu » qui ne soit imposteur, malfaisant) :

Reviens à la raison, prédicant, ton Jésus ne vaut pas mieux que Mahomet, Mahomet pas mieux que Moïse, et tous trois pas mieux que Confucius qui pourtant dicta quelques bons principes pendant que les trois autres déraisonnaient; mais en général tous ces gens-là ne sont que des imposteurs, dont le philosophe s'est moqué, que la canaille a crus et que la justice aurait dû faire pendre ${ }^{13}$.

À la question du « Quoi de neuf ? », Sacha Guitry répondait : « Molière ! » Je répondrai volontiers « Mirbeau! »

Journaliste, critique d'art, romancier et dramaturge, Octave Mirbeau (18481917) a passé son enfance dans une famille petite-bourgeoise du Perche ornais et a fait ses études secondaires au collège des jésuites de Vannes, livré par son père à ceux qu'il appellera plus tard des « pourrisseurs » ou des « pétrisseurs d'âmes », avant d'en être chassé, quelques semaines avant la fin de l'année scolaire de 1863, dans des conditions plus que suspectes, qu'il transposera dans son roman de 1890, Sébastien Roch. De ces quatre années de « véritable enfer », de cette jeunesse passée entre les mains de pseudo-éducateurs qui, en toute impunité, se livrent au « meurtre » des enfants qui leur sont confiés, Mirbeau a toujours conservé « la haine de l'éducation religieuse », comme il le rappellera dans sa réponse de 1902 à une enquête de La Revue blanche : « [...] Les maisons d'éducation religieuse, ce sont des maisons où se pratiquent ces crimes de lèse-humanité. Elles sont une honte et un danger permanent $\gg^{14}$.

Dès l'adolescence, Octave Mirbeau fut - et il le demeurera toute sa vie - un athée radical, un matérialiste impitoyablement lucide, un anticlérical absolu et un anticatholique résolu ${ }^{15}$.

12 Sade, Euvres, t. I, Paris, Éditions Gallimard, 1990, coll. « Bibliothèque de la Pléiade $\gg$, p. 5-7.

${ }^{13}$ Ibidem, p. 9.

${ }^{14}$ La Revue blanche, $1^{\mathrm{er}}$ juin 1902.

${ }^{15}$ Voir ma notice sur lui, [in] Dictionnaire des sexualités, Paris, Robert Laffont, coll. « Bouquins » (volume conçu sous l'égide de Janine Mossuz-Lavau), 2014, p. 552-554. 
Car Dieu est bel et bien mort. Nietzsche l'a proclamé en 1882. La mort de Dieu est un évènement consommé ${ }^{16}$. Pour Mirbeau, les représentations de Dieu et les mythes et légendes qui s'y rattachent ne sont que des superstitions dignes d'un « pensionnaire patenté de Charenton $\gg^{17}$ : le dieu des religions du Livre n'est qu'une « chimère », comme le proclame l'abbé Jules, inventée et instrumentalisée par les dominants pour mieux écraser les faibles, dont ils exploitent la crédulité foncière et le besoin d'espérance ${ }^{18}$.

Réfractaire à tout fanatisme, Mirbeau ne croit en aucun Dieu qui le soutienne et à nulle « Vérité suprême » qui vienne l'épauler. Il est seul. Effroyablement seul. Lucide. Terrifiquement lucide. Mais Mirbeau n'est jamais manichéen. Même Sébastien Roch n'est pas dépourvu d'ambiguités et ne saurait se réduire à un pamphlet anticlérical visant à dénoncer les crimes sexuels de prêtres catholiques couverts par leur Église et bénéficiant d'une totale impunité. Car enfin, le père de Kern, le séducteur et violeur du petit Sébastien est aussi celui qui l'initie à l'art et à la littérature et lui communique le sens de la beauté. De Kern n'est pas seulement un bourreau et Sébastien n'est pas seulement une victime.

Octave Mirbeau s'est longtemps, longuement battu, pour la séparation de l'Église et de l'État (les deux «É » majuscules, avec accent étroitement acoquinés), mais sans guère d'illusions. Il ne sert à rien en effet pour lui de laïciser les façades des écoles et des mairies si l'esprit clérical, fait de dogmatisme, de soumission à la hiérarchie et à l'abêtissement se meut en servitude volontaire.

Roger Vailland (1907-1965), lui ${ }^{19}$, avec son perpétuel goût du défi, de la provocation, fut considéré comme gênant pour les uns à cause de ses (momentanées) allégeances communistes et pour les autres à cause d'un mode de vie libertin, libertaire ${ }^{20}$. « Le général Laclos mourut à Tarente, le 5 septembre 1803, après

$16 \ll$ Dieu est mort 》 (en allemand : « Gott ist tot $\gg$ ) est une citation bien connue de Friedrich Nietzsche. Cette phrase apparaît pour la première fois sous sa plume dans Le Gai Savoir (1882), aux aphorismes 108 (《Luttes nouvelles ») et 125 (《L'insensé »), et également une troisième fois dans l'aphorisme 343 (《Notre gaieté »). Cet apophtegme se trouve aussi dans Ainsi parlait Zarathoustra et c'est surtout à cet ouvrage qu'on doit la popularité de l'expression.

17 O. Mirbeau, Correspondance générale, t. I, Lausanne, L’Âge d'homme, 2003, p. 89.

18 Le jeune Mirbeau de dix-neuf ans écrit par exemple : «C'est dans ces cérémonies religieuses que se développent dans toutes leurs formes et dans toutes leurs cruautés, l'ignorance, l'abrutissement et l'exploitation de la naïveté humaine $\gg . I b i$ dem, p. 88.

19 A. G. Leduc, Roger Vailland (1907-1965), Un homme encombrant, Paris, L'Harmattan, coll. «Socio-anthropologie » (dirigée par Pierre Bouvier), 2008.

${ }^{20}$ Voir ma notice sur Roger Vailland, [in] Dictionnaire des sexualités, p. 862-865. 
une maladie de 54 jours, sans avoir accepté les secours de la religion; tels sont les géomètres », écrit-il dans son Laclos par lui-même $e^{21}$.

《La critique de la religion est la condition préliminaire de toute critique », estime Marx, dans sa Contribution à la critique du droit de Hegel (1844). Totalement matérialiste, Roger Vailland sait que tout gain de liberté se fait par la raison, jamais par le «sacré ». Nulle Schöne Seele romantique, chez lui, cette conscience malheureuse (chrétienne) de qui a perdu son « Dieu ».

Si j’ai quelque qualité, c'est bien, je pense, d'appartenir à cette lignée essentiellement française d'esprits libres qui mène depuis des siècles le combat singulier de la Raison humaine contre la notion du sacré sous toutes ses formes, contre l'« autel et le trône », répondra-t-il à Louis Martin-Chauffier. S'il est un problème qui me soit étranger, foncièrement étranger, pas seulement intellectuellement mais physiologiquement étranger, c'est bien, me semble-t-il, le problème religieux ${ }^{22}$.

Mais pourquoi, me direz-vous, s'appesantir sur toutes ces bondieuseries, cette religiosité, ces métastases théologiques dont depuis cinq siècles une cohorte de savants, de philosophes, avait déjà fait le deuil ? Dans Le Saint-Empire (1950) Vailland charge le Vatican et les États-Unis, accusés de collusion avec les régimes fascistes. Maria, sa domestique alors qu'il vit à Capri dans la villa de son ami Malaparte (une fille de braccianti - des ouvriers agricoles - cultivant dans les Pouilles le domaine d'un prince romain), n'en revient pas de son athéisme : «Ma pieuse Maria se refuse à croire que je sois athée. Quand on ne croit pas en Dieu, plus rien ne vous distingue du chien... Or le maître de Maria ne peut pas être un chien $\gg^{23}$.

Dans mon roman Vanina Hesse, j'oppose, autour du $\ll \mathrm{o} \gg$ central, God et dog. « Facétieux Anglais, pour lesquels le chien est l'inverse de Dieu ${ }^{24}$, murmure un comparse du narrateur.

Si les « cantiques », la « messe » à Elsa, d'Aragon, son frère ennemi du Parti, ne peuvent théoriquement que rebuter Roger Vailland, celui-ci a aussi recours à des images catholiques ${ }^{25}$. Ainsi de la rêverie de Marat (dans Drôle de Jeu, 1945),

${ }^{21}$ R. Vailland, Laclos par lui-même, Paris, Éditions du Seuil, coll. «Écrivains de toujours », 1953, p. 175.

${ }_{22}$ Je ne cherche pas Dieu - La controverse entre Roger Vailland et Louis-Martin Chauffier. Texte établi par Alain (Georges) Leduc, Pantin, Éditions Le Temps des Cerises, coll. « Cahiers Roger-Vailland », 2007 (une plaquette dont j'ai assuré la préface et l'appareil critique).

${ }^{23}$ R. Vailland, Un homme encombrant..., p. 128.

${ }^{24}$ A. G. Leduc, Vanina Hesse, Paris, Éditions de La Musardine, coll. « Lectures amoureuses » (fondée par Jean-Jacques Pauvert), 2012, p. 101.

${ }^{25}$ L'irruption de la prière, de formes liturgiques furent justifiées par l'unification de la Résistance, chez Aragon, comme dans La Rose et le Réséda (1943). Ce sont des formes 
déclenchée par le nom de la station de métro « Madeleine » : « Madeleine, disais-je à Paméla, je te nomme Madeleine, sainte Marie-Madeleine... laisse couler tes cheveux sur moi, vois je suis maigre comme le Christ de la Pietà d'Avignon $\gg^{26}$.

Roger Vadim, dans D'une étoile l'autre, estimait à propos de Vailland qu'«il rejetait avec la même rigueur le puritanisme judéo-chrétien et l'hypocrisie communiste, en ce qui concernait le sexe et le droit de l'homme au plaisir $\gg^{27}$. Or, libertinage et adhésion à un parti sont incompatibles et toute transgression sexuelle va de pair avec la condamnation de la religion.

Je ne croyais être à ce point servi par l'actualité en travaillant à cette intervention : or, l'Islande légalise le blasphème. Le magazine Ijsberg nous informe que le parlement islandais vient de voter une loi pour décriminaliser le blasphème. Trois députés du Parti pirate avaient proposé ce texte suite aux attentats de janvier $2015^{28}$.

Pologne, Algérie, Russie, pour ne prendre que trois exemples, encouragent les ravages de la religion au sein de la société, avec la complicité active et les moyens de l'État. L'Église polonaise fait pression sur le gouvernement pour une interdiction totale de l'interruption volontaire de grossesse, au demeurant déjà strictement limitée et seulement autorisée dans trois cas : quand il existe un risque pour la vie ou la santé de la mère, lors d'une grave pathologie de l'embryon ou quand la grossesse est le résultat d'un viol ou d'un inceste.

En Pologne, le code pénal, interdit, je cite, de « fâcher les sentiments religieux chrétiens ». Ignacy Krasicki, un évêque polonais du XVIII siècle dont l'œuvre se moquait du mode de vie du clergé polonais de l'époque peut s'en retourner dans sa tombe...

« Le Parlement n'est pas Dieu le père ! », s'est exclamé le cardinal Philippe Barbarin, archevêque de Lyon, en septembre 2012, lors de la controverse autour du « mariage pour tous », donnant accès aux couples homosexuels au mariage (le « primat des Gaules », un des porte-parole de l'ultra-droite conservatrice, s'est vu récemment inculpé suite à son implication dans la protection de prêtres pédophiles).

Revendiquons tous en Europe le droit au blasphème, le droit de se contredire, le droit d'en rire ; tout comme Régis Debray :

auxquelles l'on peut avoir recours, en cas extrême. La lutte contre le nazisme en fut une. Le symbole de Jeanne d'Arc n'est pas épuisé, ni ceux de l'Annonciation ou de la Pietà.

${ }^{26}$ R. Vailland, Drôle de Jeu, Lausanne, Éditions Rencontre, 1967, p 74. Le roman a reçu le prix Interallié la même année.

27 Paris, Éditions n 1, 1986, p. 240.

${ }^{28}$ Libération, 3 juillet 2015. 
Les théocraties n'aiment pas le rire, les idéocraties non plus. Cela ne fait pas de la grosse rigolade notre prière du matin, mais quand un pouvoir politique censure le dessin d'humour ou le trait d'esprit à son encontre, c'est qu'il prétend occuper la place de l'Absolu. Le caricaturiste, où que ce soit, est la sonnette d'alarme ${ }^{29}$.

François Bayrou, le président du MoDem (le Mouvement démocrate, centre droit) a suggéré sur France 2 que « l'humour, la satire et l'irrévérence » soient indispensables au fonctionnement de toute démocratie et donc d'une « société comme la nôtre $\gg$. Le politicien estime que toute société a besoin d'un contre-pouvoir et « le contre-pouvoir le plus efficace, celui qui amène les puissants à réfléchir, est la satire $\gg^{30}$.

La lecture de Philippe Sollers est quasiment toujours décapante :

J'ai des amis qui me disent : « Je vais en Chine, j'emporte ma tablette, je vais lire Voltaire dans l'avion ». Mais dans l'avion, ils ont regardé le film et relu leurs mails. Étonnez-vous après qu'il y ait du fanatisme dans l'air. L'ignorance croissante, l'éradication de l'histoire à l'école, l'illettrisme galopant, la misère de la philosophie, il faut remédier à tout cela. Aujourd'hui, on parle du service civique, de réapprendre à lire, il serait temps ! Même les gens qui lisent un peu, ou qui ont lu, ou qui savaient lire, oublient qu' ils ont lu. Et la plupart de ceux qui lisent encore ne lisent que des yeux. Alors que Voltaire, vous savez, il faudrait lire chaque matin un extrait de sa correspondance, et il faudrait le lire le crayon à la main ! ${ }^{31}$

A-thée, a-gnostique - nous sommes ficelés par la sémantique -, auxquels les timorés ont accolé du négatif, le privatif, le «sans quelque chose ».

Si l'on ne mène pas d'urgence un drastique programme de laïcisation de l'école, le problème ne va aller qu'en s'aggravant. On continuera à avoir des segments de la société où la religion joue un rôle hégémonique. Le débat sur la laïcité a ressurgi voici quelques années à propos $\mathrm{du}$ 《 voile islamique », puis, l'été 2016, le burkini. Depuis les attentats de janvier contre Charlie hebdo et un magasin casher (janvier 2015); ceux simultanés en juin, en Isère, en Tunisie, au Koweït, et depuis de nouveau à Paris, à Bruxelles, à Nice, et a tant d'autres endroits (Nigeria, Burkina Faso, à Saint-Étienne-du-Rouvray, près de Rouen, où un vieux prêtre a été sauvagement égorgé), il s'est enflammé pour devenir l'un des sujets politiques majeurs, liant inextricablement les questions de la place des religions dans l'espace public, des valeurs républicaines et de la montée de l'antisémitisme, du racisme. Le « vivre-ensemble »- l'expression « exister-ensemble » serait plus judicieuse -, le lien social sont dévalorisés. Laïcité ? Quelle laïcité ? Antireli-

29 Le Monde, 27 janvier 2016, p. 13.

30 Le 2 juillet 2015, au bulletin d'information de 20 heures.

31 Ph. Sollers, op. cit. 
gieuse, inclusive, identitaire ? De quelle laïcité parle-t-on ? Le livre sur la laïcité de Jean Baubérot nous aide à identifier sept sens différents, et même parfois contradictoires, de cette référence de la France d'aujourd'huii ${ }^{32}$.

Dans un arrêt du 21 janvier 1999, la Cour Européenne des Droits de l'Homme $(\mathrm{CEDH})$ déclare :

La liberté d'expression vaut non seulement pour les informations et idées accueillies avec faveur ou considérées comme inoffensives, indifférentes, mais aussi pour celles qui heurtent, choquent ou inquiètent l'État ou une fraction quelconque de la population. Ainsi le veulent le pluralisme, la tolérance et l'esprit d'ouverture sans lesquels il n’est pas de société démocratique.

Permettez-moi encore trois citations, sur notre sujet, in fine. La première de Nasr Eddin Hodja, un alem mythique, personnage ingénu et faux-naïf prodiguant des enseignements tantôt absurdes tantôt ingénieux, qui aurait vécu en Turquie de 1208 à 1284 :

L'heure de la prière arrive au moment où Nasr Eddin atteint le bord d'une rivière. Il procède soigneusement à l'ablution rituelle et il en a presque fini avec cette obligation quand, d'un mouvement maladroit, il laisse échapper sa babouche, que le courant emporte.

Levant alors la tête vers le ciel, il crie :

« Reprends ton ablution et rends-moi ma babouche ».

Puis (anonyme) : « Vous savez pourquoi Haïfa est un modèle de tolérance en Terre sainte ? », raconte un Juif mécréant. « Parce que ni Jésus, ni Moïse, ni Mahomet, ne se sont jamais arrêtés ici ». Et enfin Léo Ferré : «Si Dieu existait, comme le disait Bakounine, ce camarade vitamine, il faudrait s'en débarrasser ».

Face au regain vindicatif des religions, il y a urgence à réhabiliter l'esprit des Lumières et d'en revenir à ce noyau dur hérité des $\mathrm{XVI}^{\mathrm{e}}, \mathrm{XVII}^{\mathrm{e}}$ et $\mathrm{XVIII}{ }^{\mathrm{e}}$ siècles européens. Adopter une attitude rigoureusement intellectuelle : esprit critique, aptitude à l'autocritique, capacité d'innovation fondée sur le droit à remettre en question toute croyance ou toute autorité instituées. En finir avec ces plaies que suscite le retour des fondamentalismes religieux, du communautarisme, du populisme et toutes les pathologies de la croyance. Prôner le retour à la raison, au savoir scientifique, à la tolérance, bref, au combat politique incessant contre l'asservissement au nom de telle ou telle religion.

32 Les 7 laïcités françaises, de Jean Baubérot, Paris, Maison des Sciences de l'homme, coll. «Interventions », 2015. 
Refusons ensemble la perpétuation de la domination des cerveaux et des corps.

Il faut plus que jamais être impolitiquement incorrect, appeler un chas un chas, une épingle une épingle.

\section{The domination of brains and bodies}

Has reading and studying Sade, Octave Mirbeau or Roger Vailland become impossible?

Are atheists, secularists, materialists now ostracized minority in Europe? It seems that in France, Ireland, Poland, Portugal, etc., henceforth, debating between the believers, the religious faithful, is only allowed. Followers and representatives of the three revealed religions, basically.

In the face of the decline of free thinking, prejudices that multiply, we must defend the heritage of the Enlightenment, their values, against obscurantism in full revival. The values of reason, knowledge, education.

The author, a careful reader of Sade, a board member of the Friends of Octave Mirbeau, creator and editor of the site Roger Vailland, will build on these three writers, decidedly atheistic and materialistic.

All three are direct descendants of the Enlightenment. Straight away for the first, the second at the turn of two centuries, the nineteenth and twentieth, the third dead here fifty years, in 1965 .

Keywords: Sade - Mirbeau - Vailland - religion - atheism

Mots-clés : Sade - Mirbeau - Vailland - religion - athéisme 